\title{
Neural Network Analysis of Nonlinear Effects of Hardiness on Burnout in Chinese Nurses
}

\author{
Felix Ladstätter ${ }^{1}$, Eva Garrosa ${ }^{2}$, Junming Dai ${ }^{3}$ \\ ${ }^{1}$ Department of Psychology, IE University, Segovia, Spain \\ ${ }^{2}$ Faculty of Psychology, UAM, Madrid, Spain \\ ${ }^{3}$ School of Public Health, Fudan University, Shanghai, China \\ Email: fladstatter@faculty.ie.edu, eva.garrosa@uam.es, imdai@fudan.edu.cn
}

Received January 2014

\begin{abstract}
Substantial research attention is evident in the hardiness and related literature concerning the topic of moderational effects of hardiness on work-related stressors and strains. In this research mostly linear methods have been used to analyze these moderational effects. However, it is not very likely that these effects are purely linear. The present study uses a neural network, a method which can model nonlinear relationships, to analyze the effects of hardiness. A cluster analysis of 268 Chinese nurses based on their self-ratings in the hardiness dimensions of commitment, challenge, and control was performed. Two groups of individuals were identified, consisting of (1) those who scored above average and (2), those who scored below average on all hardiness dimensions. On the basis of these clusters, a multi-layer neural network was used to analyze the data.
\end{abstract}

\section{Keywords}

Hardiness, Cluster Analysis, Artificial Neural Network, Burnout, Nursing

\section{Introduction}

Nursing is a profession which is highly vulnerable to stress, and it is considered that the stressors nurses are exposed to a problem that affects the practice worldwide [1]. During the past decade, the health care system has experienced major changes (e.g. rising readmission rates, the ever-growing emphasis on efficiency, etc.).

The Peoples' Republic of China (PRC) faces a unique set of challenges in health service delivery. The rapid transformation during the last 30 years in the PRC towards a modern working life is associated with increasing demands for learning new skills, the need to adapt to new types of work, pressure for higher productivity and quality of work, time pressure, hustle and growing psychological workload and stress among the workforce [2], especially in the health professions.

Burnout is understood as a specific occupational stress in human services, resulting from the psychologically and emotionally demanding relationships between caregivers and their patients. The operational definition of 
burnout in human services has three dimensions: emotional exhaustion, depersonalization, and lack of personal accomplishment. Based on these three dimensions, the experience of burnout can be alleviated by the availability of personal resources.

Ever since Kobasa [3] introduced the concept of hardiness as an important personality characteristic affecting the relationship between stressors and strains, many studies showed its relevance for health and performance [4]. Hardiness has been characterized by the three interrelated dimensions (3Cs) of commitment, control, and challenge. Extant evidence shows that hardy people perform better and stay healthier in the face of stress [5].

\section{Method}

The Nursing Burnout Scale—Short Form (NBS-SF) was used to evaluate the process of burnout. The survey offers measures of specific job stressors in nursing as antecedents of burnout (16 items), burnout (12 items), hardiness (12 items), and consequences of burnout (12 items), totaling in 52 items.

\section{Results}

Cronbach alphas were calculated to ensure the reliability of the NBS-SF scale (Table 1).

We used the k-means cluster analysis to categorize participants on the basis of their mean z-scores on each of the hardiness dimensions. Clusters that would make sense if obtained according to the existing theoretical background on the role of hardiness include patterns comprising above average or below average scores on each hardiness dimension. The mean $z$-scores of each hardiness dimension by cluster and the percentage of participants in each cluster are reported in Table 2. Cluster 1 consists of hardy individuals and cluster 2 of non-hardy individuals. To confirm that the clusters extracted from the data indeed consist of individuals with different characteristics besides their distinct hardiness profile, comparisons between clusters were conducted to determine whether or not they were higher on variables that have been proposed to be associated with hardiness, using one-way ANOVA. Mean scores on all measures for both clusters are shown in Table 1.

In order to further improve our understanding of how hardiness produces its effect and, especially, to show that the relationships between the stressors and strains, as well as the moderating effect of hardiness are nonlinear, artificial neural networks, a relatively new methodology that was found to be superior to regression in numerous problem domains [6] was used. Specifically, a three-layer feed-forward network was used for the hardiness model approximation. After training and simulation of the network, a linear regression was performed between the network outputs and the desired outputs for each of the three types of consequences, separately and combined.

To assess the predictive capacity of the burnout model, a network validation was executed after the training. All results including t-test statistics and their significance levels are summarized in Table 3 . The t-test was used to compare the predicted outcome of the neural network with the desired outcome.

Visual analyses revealed that the relationships between stressor variables (antecedents), burnout, and consequences were not only nonlinear but also different for hardy and non-hardy individuals. Figure 1 exhibits a contour plot in which physical consequences are shown as a function of work overload and contact with death and pain for hardy and non-hardy individuals.

The contour plot graphically illustrates that the stressors role ambiguity and contact with death and pain are not linearly related to organizational consequences because the surface is not a flat plane but instead a curved surface. It furthermore reveals the nonlinear influence of hardiness in this relationship because the curved surface displays different shapes for hardy and non-hardy individuals.

\section{Discussion}

The clusters we identified are suggestive of possible moderational effects on the relation observed between stressors and strains. Specifically, hardy people who score above average on all hardiness dimensions score below average on all stressors, burnout dimensions and on psychological consequences. Non-hardy individuals who score below average on all hardiness dimensions score above average on all stressors, burnout dimensions and on organizational and physical consequences (see Table 1).

The neural network analysis revealed nonlinear relations (Figure 1) between the stressors and strains as well as a moderational effect of hardiness. 
Table 1. Cronbach alphas, means and standard deviations of associates for both hardiness clusters and F-statistic of the one-way ANOVA.

\begin{tabular}{|c|c|c|c|c|c|c|}
\hline \multirow{2}{*}{ Variables } & \multirow{2}{*}{ Alpha } & \multicolumn{2}{|c|}{ Cluster 1} & \multicolumn{2}{|c|}{ Cluster 2} & \multirow{2}{*}{$\mathrm{F}(1266)$} \\
\hline & & M & SD & $\mathrm{M}$ & SD & \\
\hline \multicolumn{7}{|l|}{ Antecedents } \\
\hline Role ambiguity & 0.73 & 2.22 & 0.466 & 1.86 & 0.469 & $37.60^{* * *}$ \\
\hline Contact with death and pain & 0.75 & 2.66 & 0.449 & 2.23 & 0.479 & $43.33^{* * * *}$ \\
\hline Troubled interaction & 0.76 & 2.62 & 0.539 & 2.33 & 0.536 & $15.81^{* *}$ \\
\hline Work overload & 0.78 & 2.79 & 0.522 & 2.48 & 0.561 & $17.91^{* *}$ \\
\hline \multicolumn{7}{|l|}{ Burnout } \\
\hline Emotional exhaustion & 0.81 & 2.58 & 0.566 & 2.35 & 0.598 & $10.33^{* *}$ \\
\hline Depersonalization & 0.79 & 2.22 & 0.476 & 1.87 & 0.472 & $15.28^{* * *}$ \\
\hline Lack of personal accomplishment & 0.74 & 2.40 & 0.533 & 1.93 & 0.471 & $37.00^{* * *}$ \\
\hline \multicolumn{7}{|l|}{ Consequences } \\
\hline Psychological C. & 0.77 & 2.27 & 0.483 & 1.99 & 0.509 & $20.21^{* * *}$ \\
\hline Organizational C. & 0.85 & 2.90 & 0.540 & 2.52 & 0.570 & $31.24^{* * *}$ \\
\hline Physical C. & 0.78 & 2.74 & 0.527 & 2.51 & 0.485 & $13.60^{* * *}$ \\
\hline \multicolumn{7}{|l|}{ Hardiness } \\
\hline Commitment & 0.79 & 2.32 & 0.356 & 2.98 & 0.400 & $201.15^{* * * *}$ \\
\hline Challenge & 0.80 & 2.46 & 0.445 & 3.08 & 0.361 & $100.48^{* * *}$ \\
\hline Control & 0.83 & 2.49 & 0.373 & 3.14 & 0.308 & $179.76^{* * *}$ \\
\hline
\end{tabular}

${ }^{* *} p<0.01 .{ }^{* * *} p<0.001$.

Table 2. Number of participants per cluster. Percentage of the sample and mean z-scores for all three hardiness dimensions.

\begin{tabular}{|c|c|c|}
\hline & \multicolumn{2}{|c|}{ Cluster } \\
\hline & 1 & 2 \\
\hline$N$ & 145 & 123 \\
\hline$\%$ of total $(N=268)$ & $55.1 \%$ & $44.9 \%$ \\
\hline \multicolumn{3}{|l|}{ Hardiness dimensions } \\
\hline Commitment & 0.60 & -0.74 \\
\hline Challenge & 0.51 & -0.63 \\
\hline Control & 0.53 & -0.65 \\
\hline
\end{tabular}

Table 3. Determination coefficients $\left(R^{2}\right)$ and t-Test statistics $(t, p)$ for the neural network.

\begin{tabular}{ccccccccccc}
\hline Data sets & \multicolumn{3}{c}{ Psychological } & \multicolumn{3}{c}{ Organizational } & \multicolumn{3}{c}{ Physical } \\
\hline & $R^{2}$ & $t$ & $p$ & $R^{2}$ & $t$ & $p$ & $R^{2}$ & $t$ & $p$ \\
Training $(N=227, d f=226)$ & 0.57 & 0.305 & 0.698 & 0.67 & 1.197 & 0.263 & 0.49 & 0.299 & 0.765 \\
Validation $(N=49, d f=48)$ & 0.37 & -1.361 & 0.225 & 0.39 & 0.646 & 0.521 & 0.36 & -0.889 & 0.394 \\
\hline
\end{tabular}

For both, hardy and non-hardy individuals, there is a relatively flat area for contact with death and pain levels of 1 - 3 and work overload levels of 1 - 3. This tells us that within this area, even if the input variables change within the above mentioned ranges, psychological consequences keep constant (and low). However, if the levels of contact with pain and death increase (3 - 4) we find a very steep area which indicates that in this area the variable contact with pain and death has a severe effect on psychological consequences, namely, they increase dramatically especially in the case of non-hardy persons. This means that there is a point in the range of contact 

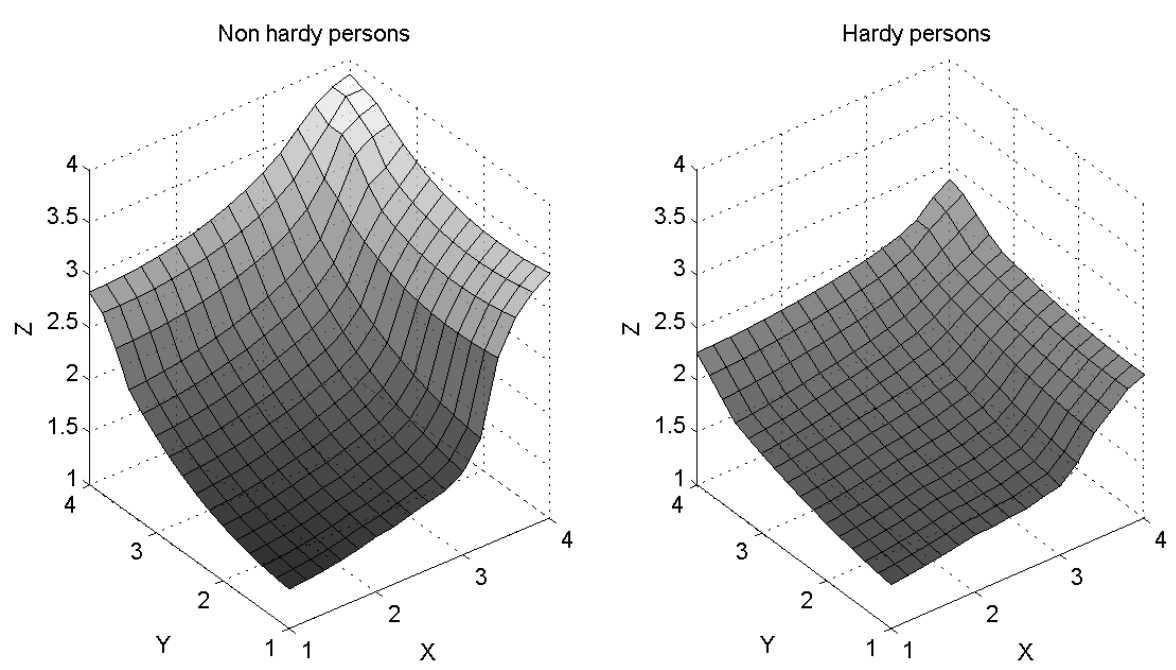

$\mathrm{X}$ : Contact with pain and death

Y: Work overload

Z: Psychological consequences

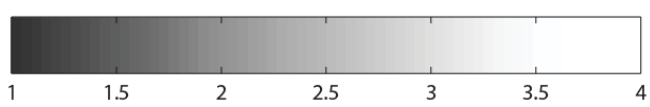

Figure 1. Effect of Hardiness on the Relation between Stressors and Strains.

with pain and death levels until which change has little to no effect on psychological consequences. However, if this point is exceeded, the psychological consequences increase severely.

An interesting issue for further research would be if other hardiness profiles besides hardy and non-hardy ones might deepen the understanding of how hardiness affects the relation between stressors and strains.

\section{References}

[1] Schaufeli, W.B. and Enzmann, D. (1998) The Burnout Companion to Study and Practice: A Critical Analysis. Taylor \& Francis, London.

[2] Garrosa, E., Moreno-Jiménez, B., Ladstätter, F. and Liang, Y. (2006) Globalization of Occupational Health: China’s Development and Reform. Medicina y Seguridad del Trabajo, 202, 39-44.

[3] Kobasa, S.C. (1979) Stressful Life Events, Personality, and Health: An Inquiry into Hardiness. Journal of Personality and Social Psychology, 37, 1-11. http://dx.doi.org/10.1037/0022-3514.37.1.1

[4] Eschleman, K.J., Bowling, N.A. and Alarcon, G.M. (2010) A Meta-Analytic Examination of Hardiness. International Journal of Stress Management, 17, 277-307. http://dx.doi.org/10.1037/a0020476

[5] Bartone, P.T., Eid, J., Johnsen, B.H., Laberg, J.C. and Snook, S.A. (2009) Big Five Personality Factors, Hardiness, and Social Judgment as Predictors of Leader Performance. Leadership and Organization Development Journal, 30, 498521. http://dx.doi.org/10.1108/01437730910981908

[6] Ladstätter, F., Garrosa, E., Badea, C. and Moreno, B. (2010) Application of Artificial Neural Networks to a Study of Nursing Burnout. Ergonomics, 53, 1085-1096. http://dx.doi.org/10.1080/00140139.2010.502251 